\title{
COMENTARIO EDITORIAL: Infecciones respiratorias agudas en niños: costo para las familias y utilización de servicios
}

\author{
Acute respiratory infections in children: cost for families and resource utilization
}

Javier Vilosio*

\begin{abstract}
Resumen
Se comenta un artículo publicado recientemente en el que se estiman los costos que implica para las familias la internación de un niño por infección respiratoria aguda baja (IRAB) se comparan los mismos entre un centro de tercer nivel de atención (Hospital Garrahan) y otros de menor complejidad ubicados en la provincia de Buenos Aires, y se exploran los motivos por los que la población, aún debiendo afrontar mayores gastos, consulta fuera de su área programática. Se resalta la escasez de antecedentes respecto de este tipo de investigaciones en Argentina, y su importancia, dada la relevancia sanitaria y social del problema.

El carácter catastrófico de un episodio de internación por IRAB para las familias analizadas, hasta el 80\% pobres e indigentes, se manifiesta en el hallazgo de que cada episodio (de aproximadamente 8,5 días de duración) implica un gasto de 20 a $40 \%$ de los ingresos totales del grupo familiar, afectando en mayor grado a los indigentes.

Se discuten algunos aspectos que hacen a la orientación espontánea de la demanda por fuera de las redes sanitarias previstas. Se comenta críticamente que el desarrollo de tales redes suele depender más de consideraciones políticas y territoriales, que de concepciones funcionales.

Se enfatiza en la necesidad de promover análisis de este tipo, como basamento para la planificación y el desarrollo de proceso de adecuación de la oferta a las necesidades de la población.

\section{Abstract}

This editorial comments on a recently published work about estimated cost for families related to hospitalisation of children with acute lower respiratory infection (ALRI) according to complexity and location of hospital. It also explores family reasons for attending hospitals outside their programmatic area, even at risk of higher cost. It also refers to the shortage of local cost related information and its importance in relation to sanitary and social issues. The catastrophic characteristics of an episode of acute lower respiratory infection related hospitalisation for the families in the study, $80 \%$ of which were of low socio-economic background, are exemplified by the fact that $20-40 \%$ of total family income were spent during a single episode, this affecting in a higher degree those families with lowest socio-economic situation.

Another aspects discussed here are related to spontaneous demand orientation outside provided local sanitary network. These networks usually rely on political and territorial considerations rather than functionality issues. The author states the need of this type of economic analysis for planning and developing adequacy of health services offer in relation to population needs.
\end{abstract}

Palabras clave: infección respiratoria aguda-costos-niños-familias. Key words: acute respiratory infection-costs-children-families.

Vilosio J. Infecciones respiratorias agudas en niños: costo para las las familias y utilización de servicios. Evid Act Pract Ambul. 11(1). 4-6. Ene-Feb 2008.

\section{Intoducción}

Promediando el invierno de 2007, se informó que sólo en la Provincia de Buenos de Aires se habían registrado unos 10.000 casos más de infecciones respiratorias agudas en los niños que en 2006.

Las autoridades sanitarias nacionales y provinciales atribuyen estos números a la implementación exitosa de un programa de contingencia establecido, que habría permitido mejorar la detección precoz de los casos e implementar un adecuado tratamiento, tal y como se refleja en la menor incidencia este año de casos graves y defunciones.

Dado que todavía no se dispone de información adecuada para precisar los motivos reales de tal incremento en la demanda ni para evaluar la efectividad del programa implementado, debemos considerar también las características particularmente duras del invierno pasado actuando sobre una vasta población en riesgo socio-económico como causas del desborde de la capacidad asistencial disponible, según fue ampliamente difundido por los medios de comunicación.

En Febrero de 2007 se publicó en los Archivos Argentinos de Pediatría, un artículo titulado Carga de enfermedad y costos asociados a las internaciones por infección respiratoria aguda en niños 1 cuyos objetivos fueron estimar el costo que implica para las familias la internación de un niño por infección respiratoria aguda baja (IRAB) y realizar una evaluación económica comparativa de los costos asociados a la internación entre un centro de tercer nivel de atención y los centros de menor com- plejidad próximos al domicilio, así como explorar los motivos que llevan a la población a consultar fuera de su área programática.

Para ello se compararon datos de 384 pacientes internados durante el invierno de 2004 en un grupo de hospitales de la Provincia de Buenos Aires y en el Hospital Nacional de Pediatría "Profesor Dr. Juan. P. Garrahan" de la Ciudad Autónoma de Buenos Aires.

Los autores del trabajo comentan que los estudios referidos a costos directos e indirectos de las IRAB son escasos en la bibliografía existente. Agregamos nosotros que esto es así no sólo en lo referidos a esta patología, sino que se trata de un tipo de estudio infrecuente en la producción nacional. Por otra parte, dentro de la relativamente escasa producción argentina respecto de la economía sanitaria, la evaluación de los costos de la enfermedad para las personas y las familias, constituye un área particularmente inexplorada. En principio, la dificultad para la obtención de datos útiles, sumada a la escasez de financiamiento disponible para este tipo de estudios ${ }^{\S}$, y la percepción generalizada de que esta información no constituye un insumo usualmente requerido para la toma de decisiones sanitarias, podrían explicar al menos parcialmente esta situación. El trabajo que comentamos tiene el doble mérito de sumar a los hallazgos referidos a sus objetivos específicos, el de servir para plantear una discusión sobre el tipo de investigaciones que interesan a los servicios de salud, sus posibilidades, limitaciones y requerimientos, y la racionalidad subyacente a las decisiones en el campo de la gestión y las política sanitarias. 


\section{El problema sanitario, un problema social}

Las IRAB constituyen, ciertamente, un problema de la salud pública. Proyectando los números utilizados en el trabajo que comentamos, podríamos establecer que en Argentina, para una cohorte de 750.000 nacimientos anuales, alrededor de 1.800 .000 niños -450.000 menores de un año- sufrirían al menos un episodio de infección respiratoria aguda, cada año. De ese total, alrededor de 270.000 requerirían tratamiento en un servicio de urgencias pediátricas, y unos 7.500 lactantes necesitarían ser internados por IRAB.

Este año, de acuerdo a los datos disponibles en el momento de redactar este comentario, en la provincia de Buenos Aires y hasta la semana epidemiológica (SE) número 26 se registró un aumento del $4 \%$ de los casos de bronquiolitis en niños menores de un año y del $10 \%$ en niños de uno a dos años, mientras las neumonías se incrementaron un $12 \%$ respecto de $2006^{2}$.

En la ciudad de Buenos Aires fueron informados durante el mismo período, 19.901 casos de bronquiolitis y 7.131 de neumonías en niños. Un aspecto muy importante del informe provisto por el Gobierno de la Ciudad de Buenos Aires (GCBA) es que el $52 \%$ de las bronquiolitis y el $18 \%$ de las neumonías informadas corresponden a niños domiciliados en la provincia de Buenos Aires

Un excelente trabajo presentado por Bossio ${ }^{4}$ nos permite además conocer cómo se distribuyen las IRAB en relación con ciertas variables, fundamentalmente sociales y económicas, lo cual define el grupo poblacional sobre el que impactan los hallazgos obtenidos por Rowenztein y col.

El bajo peso al nacer, el analfabetismo materno y el ser hijo de madre adolescente, aumentan entre tres y cinco veces el ries go de morir durante en el primer año de vida por enfermedades respiratorias. Como es bien sabido, las diferencias entre distintas regiones o zonas geográficas ${ }^{* *}$ que impactan sobre la salud son las condiciones económicas y sociales, una de cuyas expresiones es también la oferta de recursos sanitarios, en disponibilidad y calidad.

\section{Un problema catastrófico}

En salud pública se definen como enfermedades catastróficas aquellas habitualmente de baja incidencia, que requieren tratamientos de alto costo y/o de prolongada cobertura en e tiempo, que ponen en riesgo vital y/o funcional al paciente y cuyo tratamiento, por lo general de alta complejidad, requiere de infraestructura, equipamiento y/o recurso humano especializado ${ }^{5}$. El problema del alto costo suele enfocarse desde el punto de vista de los financiadores ${ }^{\dagger}$, siendo menos difundido el enfoque hacia la problemática de los pacientes y sus familias.

En el caso de las IRAB, la mayoría las condiciones vinculadas a la alta complejidad pueden evitarse aplicando estrategias preventivas y de atención primaria para resolver el problema en sus estadios iniciales, evitando así los altos costos de internación de casos graves y que en los países desarrollados se ha podido reducir drásticamente ${ }^{6}$. Sin embargo en Argentina, la principal causa de egresos hospitalarios en menores de cinco años sigue relacionada a patología respiratoria.

Desde la perspectiva de los costos para la familias de estos niños, tal y como muestra el trabajo que comentamos, un episodio de internación (aproximadamente 8,5 días) significa un gasto del 20 al $40 \%$ de los ingresos totales del grupo familiar, dependiendo fundamentalmente de que ambos padres trabajen o no -peor cuando ambos lo hacen-y de la existencia de hermanos del paciente -peor cuando los hay.

Por otra parte, en la población relevada, hasta el $80 \%$ correspondió a pobres e indigentes (dependiendo del hospital donde se atendieron). Los indigentes registraron los porcentajes más elevados de pérdida de ingresos. Es decir, sufrieron el mayor costo. La contundencia de los hallazgos reportados exime de mayores comentarios respecto de la situación desastrosa que significa para la economía de las familias pobres una internación por IRAB, a la vez que nos recuerda una perspectiva diferente del concepto de enfermedad catastrófica. Una perspectiva especialmente relevante para la planificación de políticas sanitarias.

\section{Redes poco articuladas}

El concepto de referencia y contrareferencia, es decir, la articulación sistematizada de los distintos niveles del sistema de salud, constituye en sí mismo un componente fundamental de la estrategia de atención primaria, así como, en general, de la organización eficaz y eficiente de los servicios sanitarios.

Como hemos señalado, el trabajo que comentamos se propone también explorar los motivos por los cuales numerosas familias consultan por fuera de las redes sanitarias previstas en su jurisdicción de residencia y concurren directamente a un hospital especializado.

Los autores encontraron que el $55 \%$ de los pacientes lo hicieron por "falta de confianza en los centros de atención de sus áreas programáticas y confianza en el Garrahan". Pero además otro $42 \%$ lo hizo por recomendación de terceros, para quienes, podríamos asumir, el factor confianza en el hospital resultó también determinante. Como consecuencia de ello, el $97 \%$ de los pacientes con diagnóstico de IRAB ingresaron al Hospital Garrahan en forma espontánea, no derivados.

Hemos mencionado más arriba el volumen de pacientes con domicilio en la provincia de Buenos Aires que se reportan ingresados en hospitales de la Ciudad. Obviamente cuando el comportamiento de una población no se corresponde con la expectativa de los planificadores se impone la necesidad de un análisis critico de la planificación, y de los supuestos en los que la misma se basa. Algunas obviedades que a veces se ignoran son: las personas no se atienen a las normativas administrativas y jurisdiccionales, buscan atención donde creen que van a ser mejor atendidas, y esa elección es siempre la mejor posible en el marco de la información, los hábitos y los valores imperantes. La verdadera capacidad de respuesta de un sistema de servicios de salud frente a la demanda -que en el caso de las IRAB resulta razonablemente previsible en tiempo y cantidad depende no sólo de la imprescindible existencia física de los servicios, el personal y los insumos requeridos, sino que fundamentalmente se vincula con las condiciones de accesibilidad, en un sentido amplio -muchas de las cuales en realidad no

${ }^{* *}$ Para un niño argentino nacido con bajo peso y con una madre analfabeta o con ciclo primario incompleto, el riesgo de morir por una enfermedad respiratoria durante el primer año de vida es hasta 17 veces más alto, dependiendo de la jurisdicción de residencia de la madre. Los 190 departamentos con las tasas más bajas de mortalidad por enfermedades respiratorias en menores de cinco años aportan el $20 \%$ de los nacidos vivos en total y el $5 \%$ de las muertes por estas causas, mientras que los 118 con las tasas de mortalidad más altas aportan el $20 \%$ de los nacidos vivos y el $40 \%$ de las muertes.

${ }^{\dagger}$ En estos casos se suele resaltar el riesgo de quebranto al que se exponen particularmente aquellas instituciones de salud (ej. organizaciones de la seguridad social o la medicina prepaga) que no cuentan con adecuado número y distribución por riesgo de sus beneficiarios. Este es el principal fundamento de los mecanismos de redistribución a través de fondos especiales en la seguridad social, cuyo principal exponente es la Administración de Programas Especiales. 
pueden ser modificadas desde el sector salud- de condiciones de calidad de atención, incluyendo la motivación y capacitación de todo el equipo, profesional o no, del grado de consideración que se haya dado a cuestiones tales como hábitos, necesidades y demandas de la población, y a la forma en que se transmiten a ésta cuestiones tales como la disposición, organización y seguridades brindadas por los servicios.

Mas allá de la retórica habitual del sanitarismo, y reconociendo que ambos términos no guardan relación de dependencia entre sí, debemos reconocer que la idea de complejidad se asocia en muchas oportunidades fundadamente, al menos desde la óptica del usuario, con la existencia de mayores garantías de calidad en la atención.

Por otra parte, si bien la pertenencia de diversos efectores a una sola autoridad jurisdiccional tampoco garantiza su fluida integración -tal como lo demuestra la experiencia en numerosas provincias- resulta razonable suponer que la diversidad jurisdiccional complica aún más la posibilidad de articular los recursos disponibles en la referencia y contrarreferencia fortaleciendo el primer nivel de atención.

No hay duda de que el desarrollo de la infraestructura sanitaria suele depender más de consideraciones políticas y territoriales, que del concepto de funcionalidad de un sistema integrado. Lo cual es esperable en un sistema de salud atomizado como e nuestro. Sigue pendiente entonces la decisión política de integrar la Región Metropolitana desde el punto de vista de la planificación y la operación de la red de servicios existentes, y por lo tanto también la multiplicidad de necesarias modificaciones en la gestión de los servicios que creen la suficiente confianza en el público.

Por otra parte, este tema ha salido dramáticamente a la luz en días recientes, a partir de una propuesta del Gobierno de la Ciudad de Buenos Aires en el sentido de priorizar en sus hos- pitales la atención de habitantes de la ciudad, que despertó fuerte polémica y rechazos. Sin embargo, desde el punto de vista sanitario es imprescindible avanzar sobre los múltiples aspectos que hacen a la integración entre las dos jurisdicciones más importantes del país. Sería una atrocidad reducir la discusión al respecto a un mero intercambio de presiones políticas del tipo "pacientes en la Ciudad por basura en el Conurbano", tal como parece estar planteado hasta el momento.

$Y$, claro, ello implicará avanzar sobre cuestiones de infraestructura, disponibilidad de insumos y condiciones de trabajo, pero sin duda también sobre los costos y el financiamiento del sistema, las necesidades y demandas de los usuarios, y la capacitación, el entrenamiento y la motivación del equipo de salud.

\section{Hacia adelante}

Señalamos antes la originalidad del enfoque en el costo para las familias de un episodio de IRAB, y los escasísimos antecedentes de este tipo de estudios. Las conclusiones obtenidas en el trabajo indudablemente nos brindan una perspectiva inusual sobre patologías de amplia incidencia, fácilmente prevenibles en el primer nivel de atención y que terminan constituyendo verdaderas catástrofes económicas para las familias. El ya clásico modelo de ciclo económico de la enfermedad nos facilita además, la comprensión de cómo se amplifican diversas consecuencias sanitarias y sociales de la enfermedad; y la relevancia de sus costos directos e indirectos para las familias y la comunidad.

Es evidente que debiéramos promover este tipo de análisis, no sólo como ejercicios de la inteligencia sanitaria, sino como insumos imprescindibles para la planificación, y el desarrollo del constante proceso de adecuación de la oferta a las necesidades de la población. Es decir, para el desarrollo de la política sanitaria.

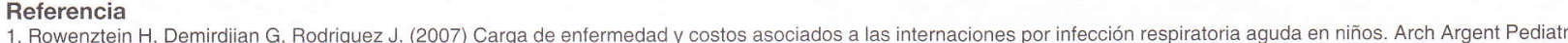
2007; 105(1):5-11

2. Ministerio de Salud Gobierno de la Provincia de Buenos Aires (2007) Infecciones Respiratorias Agudas. Enfermedad Tipo Influenza - Bronquiolitis - Neumonías. Situación 2. Ministerio de Salud Gobierno de la Provincia de

Actual, 13 de Julio 2007. Disponible al 12/08/07 en:

http://www.msal.gov.ar/htm/site/sala_situacion/PANELES/Panel_Tematico/Inf_Respiratorias_Agudas_Prov_de_Bs_As.pdf
3. Ministerio de Salud. Gobierno de la Ciudad de Buenos Aires (2007). Sala de Situación 2007. Informe disponible al 12/08/07 en:

3. Ministerio de Salud. Gobierno de la Ciudad de Buenos Aires (2007). Sala de Situación 2007. Informe disponible
http://www.msal.gov.ar/htm/site/sala_situacion/PANELES/Panel_Tematico/sala_IRA_cap_federal_03_08_07.pdf

4. Bossio, J C, Epidemiología de las IRAB (2006). Presentación en el $34^{\circ}$ Congreso Argentino de Pediatría, Sociedad Argentina de Pediatría. Córdoba, Octubre 2006.

5. Zanarini, E. (2003). Financiación de enfermedades catastró́ticas. Disponible al 13/08/07 en

http://cdi.mecon.gov.ar/biblio/docelec/MU2486.pdf

6. Dalamon R, Asnaghi P, Biedak E. (1999). Egresos por enfermedad respiratoria en lactantes y niños en Hospitales de la Ciudad de Buenos Aires. Arch Argent Pediatr 1999 $97(4): 227-235$

\section{INFO - EVIDENCIA}

\section{¿Qué es Evidencia, Actualización en la Práctica Ambulatoria?}

Una publicación independiente editada cada dos meses desde 1997 por la Fundación MF, organización sin fines de lucro dedicada a promover el desarrollo de la medicina familiar y la atención primaria de la salud.

\section{¿Cuál es el objetivo de Evidencia?}

Contribuir a la educación continua y a la actualización de los profesionales de la salud de la región en el área de la atención ambulatoria.

\section{Contenidos de Evidencia}

- Información independiente, actualizada y resumida en forma sencilla y en castellano sobre temas relevantes de la atención ambulatoria.

- Artículos seleccionados por su calidad y relevancia clínica, resumidos y comentados críticamente por destacados profesionales del área.

- Revisiones sobre temas clínicos, epidemiológicos, sanitarios o humanísticos, de importancia para la práctica ambulatoria.

- Notas farmacológicas para la actualización rápida de temas de terapéutica clínica.

- Discusión de casos clínicos con herramientas de Medicina Basada en la Evidencia.

- Glosario de términos de epidemiología y medicina basada en la evidencia

¿Cómo es posible acceder a Evidencia?

Por suscripción a la edición en papel, o bien por Internet, en nuestro sitio: http://www.evidencia.org 